\title{
Designs II Subtest (WMS-IV)
}

National Cancer Institute

\section{Source}

National Cancer Institute. Designs II Subtest (WMS-IV). NCI Thesaurus. Code C120347.

A subtest of the Wechsler Memory Scale, 4th Edition that evaluates delayed recall as well as delayed recognition. The subject is shown a grid with 4-8 designs on a page for 10 seconds, which is then removed from view. After a delay, the subject is asked to recreate the pages shown in the immediate condition. Then, the subject is shown a series of grids and asked to select the two designs that are correct and in the same place as on the page shown in the immediate condition. 